\title{
Bedarfslagen in der häuslichen Pflege
}

Andreas Büscher

4.1 Einleitung - 56

4.2 Charakteristika häuslicher Pflegearrangements - 57

4.3 Weiterentwicklung der Leistungen

der Pflegeversicherung - 59

4.4 Erwartungen an zukünftige Unterstützungsmöglichkeiten durch die Pflegeversicherung - 61

Literatur - 63 


\section{- Zusammenfassung}

Ein erklärtes Ziel der Pflegeversicherung besteht in der Priorität der häuslichen gegenüber der stationären Pflege. Um dieses Ziel zu erreichen, sieht die Pflegeversicherung eine Reihe von Leistungen vor, die in Ergänzung der Pflege durch Angehörige den Verbleib des pflegebedürftigen Menschen in der häuslichen Umgebung ermöglichen sollen. Die Betrachtung einiger Charakteristiken häuslicher Pflegearrangements verdeutlicht, dass die Bedarfslagen oftmals komplexer sind als die vorgesehenen Leistungen und diese nur für Teile davon angemessen erscheinen. Veränderungen und Erweiterungen des Leistungsspektrums der Pflegeversicherung im Laufe der Jahre verdeutlichen das Bemühen die Leistungen weiterzuentwickeln. Die Bedarfslagen in häuslichen Pflegearrangements erfordern jedoch weitere Anpassungen, auch durch die Einführung des neuen Pflegebedürftigkeitsbegriffs, die abschließend in diesem Beitrag skizziert werden.

One of the main aims of the German long-term care insurance was the promotion of home care $v s$. institutional care. In order to achieve this objective, a range of benefits was introduced which in addition to informal care provided by family members are intended to ensure the care recipients' stay in his or her own home for as long as possible. A closer look into characteristics of home care arrangements reveals that the needs are often more complex than the benefits provided and that these only cover some of the existing needs. Expansions and changes of the range of LTC benefits over the past years indicate political efforts to continuously improve the benefit scheme. Home care needs, however, require some more adjustments, particularly due to the new eligibility criteria for long-term care that are outlined at the end of this chapter.

\subsection{Einleitung}

Der Blick in die alle zwei Jahre veröffentlichte Pflegestatistik verdeutlicht seit langem die hohe Bedeutung und den hohen Anteil der häus- lichen Pflege an der Gesamtbewältigung der Pflegebedürftigkeit. 2,65 Mio. Menschen (76 \% aller pflegebedürftigen Personen) erhalten eine Unterstützung in ihrer häuslichen Umgebung, 1,76 Mio. erhalten Geldleistungen aus der Pflegeversicherung und in 830.000 Haushalten sind ambulante Pflegedienste in die Versorgung eingebunden und leisten diese zu einem unterschiedlich großen Anteil (Statistisches Bundesamt 2018). Unter häuslicher Pflege wird im vorliegenden Beitrag die Gesamtheit der pflegerischen Unterstützung in der häuslichen Umgebung eines pflegebedürftigen Menschen verstanden. Diese kann durch Angehörige, Freunde, Bekannte oder andere Personen im Rahmen ihrer sozialen Beziehung zum pflegebedürftigen Menschen erfolgen. Diese Unterstützung wird oftmals auch als informelle Pflege bezeichnet. Unter formeller Pflege wird demgegenüber die Unterstützung durch professionelle Leistungserbringer, insbesondere ambulante Pflegedienste, verstanden.

Zum Zeitpunkt der Einführung der Pflegeversicherung war eine der großen Befürchtungen ein Rückgang der häuslichen Pflege durch eine mögliche nachlassende Pflegebereitschaft (Meyer 1996) oder ein nachlassendes informelles Pflegepotenzial durch veränderte Erwerbsbiografien von Frauen, Auswirkungen des demographischen Wandels oder andere Entwicklungen. Die Pflegestatistik zeigt eindeutig, dass es zu diesen Entwicklungen nicht gekommen ist. Stattdessen hat sich die Anzahl der Haushalte, in denen pflegebedürftige Menschen ohne professionelle Unterstützung versorgt werden, von einer Million im Jahr 2001 (Statistisches Bundesamt 2003) auf 2,65 Mio. deutlich mehr als verdoppelt. Es kann nicht vorausgesagt werden, ob diese Entwicklung auch in den folgenden Jahren bestehen bleibt, aber es gibt keine Anzeichen, dass kurz- und mittelfristig mit einer deutlichen Trendänderung zu rechnen ist. Zwar werden demographische Entwicklungen, nach denen wenige jüngere immer mehr älteren Menschen gegenüberstehen, Auswirkungen auf die informelle Pflege haben, daraus kann jedoch nicht geschlossen werden, dass die häusliche Pflege durch Familie, Freunde und 
Bekannte bald endet. Entsprechend bleibt die Unterstützung häuslicher Pflegearrangements eine wichtige Herausforderung der Pflegeversicherung.

Die Unterstützung der häuslichen Pflege war ein erklärtes Ziel der Einführung der Pflegeversicherung und es wurden verschiedene Leistungen und Unterstützungsmöglichkeiten zur Verfügung gestellt, um dieses Ziel zu erreichen. Neben den Hauptleistungen der Pflegeversicherung, der Sachleistung für die Unterstützung durch einen Pflegedienst und der Geldleistung für selbst beschaffte Pflegehilfen, waren Leistungen zur Sozialversicherung für Angehörige und Angebote zur Teilnahme an Pflegekursen unmittelbar nach Einführung der Pflegeversicherung bereits verfügbar. Im Laufe der Jahre sind verschiedene weitere Leistungen hinzugekommen, insbesondere hinsichtlich der Beratung zu Pflegefragen. Diese Erweiterungen der verfügbaren Unterstützungsmöglichkeiten im häuslichen Umfeld sind einerseits Ausdruck der Lern- und Weiterentwicklungsfähigkeit der Pflegeversicherung. Sie sind andererseits aber auch Ausdruck vormals verkürzter Annahmen über die Wirkungen des verfügbaren Leistungsspektrums und seiner Angemessenheit angesichts vielfältiger und heterogener Bedarfslagen. Bevor im weiteren Verlauf dieses Beitrags die Leistungen eingehender hinsichtlich ihres Nutzens und der Notwendigkeit ihrer Weiterentwicklung beleuchtet werden, folgen zunächst einige Ausführungen zu häuslichen Pflegearrangements. Sie bilden den Hintergrund für die Analyse und Bewertung der verfügbaren Unterstützungsoptionen der Pflegeversicherung, insbesondere auch nach der Einführung des neuen Pflegebedürftigkeitsbegriffs zum 01.01.2017.

\subsection{Charakteristika häuslicher Pflegearrangements}

Ein häusliches Pflegearrangement entsteht in der Regel über zwei unterschiedliche Wege. Zum einen kann es sich als schleichender
Prozess entwickeln, indem zu Beginn unterschiedliche Formen der Hilfestellung und Unterstützung zwischen Familienmitgliedern geleistet werden, die sich zunehmend von einer gegenseitigen $\mathrm{zu}$ einer einseitigen Unterstützung entwickeln, da ein Mitglied des Haushalts krankheits-, alters- oder fähigkeitsbedingt in stärkerem Maß der Unterstützung bedarf. Bei einem fortschreitenden Bedarf an dieser Art der Unterstützung entsteht ein dauerhaftes Hilfsarrangement, in dem es mehr oder weniger offen zu Festlegungen kommt, wer die Hauptunterstützung leistet und wer Entscheidungen trifft.

Der zweite Weg zur Entwicklung eines häuslichen Pflegearrangements entsteht aufgrund von akuten Ereignissen wie Unfällen oder nach einem Schlaganfall. Dabei entsteht aus einem vormals etablierten Alltag innerhalb eines Haushalts unmittelbar eine kritische Situation, in der ein Familienmitglied krankheitsbedingt seinen Alltag und die darin vorkommenden Aufgaben nicht mehr bewältigen kann und umfassender Unterstützung in vielen Aktivitäten und Lebensbereichen bedarf. Die Situation, auf die nur wenige Menschen vorbereitet sind, erfordert unmittelbares Handeln und Entscheiden. In beiden Fällen besteht ein hoher Beratungsbedarf, um Entscheidungen und Vorkehrungen treffen zu können, wie mit der Situation umzugehen ist. Die Frage, ob formelle Unterstützungsangebote in Anspruch genommen werden sollen, und falls ja, welche, spielt eine wichtige Rolle.

Ausgangspunkt eines Pflegearrangements ist in beiden Fällen, dass die Selbständigkeit eines Menschen beeinträchtigt ist, der aus diesem Grund der Hilfe und Unterstützung anderer bedarf. Mit der Pflegeversicherung wurde ein Begriff der Pflegebedürftigkeit eingeführt, um den Kreis der leistungsberechtigten Personen zu definieren. Nach diesem Begriff bestand Pflegebedürftigkeit im Zeitaufwand und in der Häufigkeit für die Unterstützung bei Alltagsverrichtungen in den Bereichen Körperpflege, Ernährung, Mobilität und hauswirtschaftliche Versorgung. Dieser Begriff der Pflegebedürftigkeit wurde im Laufe der Jahre nicht nur zur 
Bestimmung des leistungsberechtigten Personenkreises genutzt, sondern war auch maßgebend für die wesentlichen Leistungen der Pflegeversicherung, die sich sehr stark an diesem verrichtungsorientierten Verständnis orientierten. Erst mehr als zwanzig Jahre nach Einführung der Pflegeversicherung kam es zu einer grundlegenden Reform dieses Begriffs. Seit dem 01.01.2017 wird Pflegebedürftigkeit als Beeinträchtigung der individuellen Selbständigkeit und das daraus resultierende Angewiesensein auf personelle Hilfe in den Aktivitäten und Lebensbereichen Mobilität, kognitive und kommunikative Fähigkeiten, Verhaltensweisen und psychische Problemlagen, Selbstversorgung, krankheits- und therapiebedingte Anforderungen und Belastungen sowie Gestaltung des Alltagslebens und sozialer Kontakte verstanden.

Dass ein auf Alltagsverrichtungen reduziertes Verständnis von Pflegebedürftigkeit und darauf ausgerichteter Leistungen nur bedingt stabilisierende Wirkung in häuslichen Pflegearrangements zu entfalten vermag, ist darauf zurückzuführen, dass die Unterstützung bei Alltagsverrichtungen zwar einen relevanten Teil der notwendigen Hilfen ausmacht, in ihrer Bedeutung jedoch hinter anderen Aspekten zurücksteht. Bereits Ende der 1980er Jahre entwickelte Bowers (1987) eine Typologie der Pflege durch Angehörige, nach der diese nicht anhand von Einzeltätigkeiten, sondern bezogen auf die dahinterstehenden Absichten beschrieben wurde. Sie unterschied:

- antizipierende Pflege, bei der es um die Antizipation von gesundheitlichen Anforderungen und der Vorbereitung vorsorglicher Maßnahmen geht,

- präventive Pflege, die sicherstellende und gewährleistende Aufgaben, z. B. bezogen auf Diäten oder Medikamente, umfasst,

- beaufsichtigende Pflege, die als Folge der präventiven Pflege notwendig werden kann, wenn diese allein nicht ausreicht,

- instrumentelle Pflege, die am ehesten mit der Unterstützung bei Alltagsverrichtungen verglichen werden kann,
- schützende Pflege, die als am schwierigsten bezeichnet wurde und bei der es um die Erhaltung des Selbstwertgefühls und der Autonomie des pflegebedürftigen Menschen geht.

Später fügten Bowers (1988) sowie Nolan et al. (1996) noch die erhaltende und wiederherstellende Pflege sowie die reziproke - also auf Gegenseitigkeit beruhende - Pflege hinzu.

Die vor allem auf instrumentelle Aspekte ausgerichteten Leistungen der Pflegeversicherung treffen nur einen Teil der hier von Angehörigen beschriebenen Pflege. Auch die ebenfalls bereits vor längerem von Twigg und Atkin (1994) vorgenommene Typologisierung pflegender Angehöriger gibt Hinweise auf umfassendere Bedarfslagen. In diesem Ansatz werden Angehörige anhand demographischer Merkmale wie Alter oder Geschlecht, anhand der Erkrankung oder Problemlage des zu pflegenden Familienmitglieds (z. B. Angehörige von Menschen mit Demenz) oder anhand der Beziehung zum pflegebedürftigen Menschen (z. B. Eltern, Kindern, Partner) charakterisiert. Für die Frage der Angemessenheit von Leistungen und der Weiterentwicklung von Unterstützungsmöglichkeiten ist dieser Ansatz von Interesse, weil sich aus jeder Betrachtungsweise unterschiedliche Aussagen zu bestehenden Bedarfslagen ableiten lassen.

Werden Angehörige unter dem demographischen Merkmal des Alters betrachtet, ist es einleuchtend, dass diejenigen im erwerbsfähigen Alter mit anderen Aufgaben hinsichtlich der Vereinbarkeit von Erwerbstätigkeit und Pflege konfrontiert sind als Angehörige, die das gesetzlich vorgesehene Rentenalter bereits erreicht haben. Für sie stellen sich sehr existenzielle Fragen zur Alterssicherung und zu den Auswirkungen der häuslichen Pflegetätigkeit darauf. Bei Angehörigen jenseits der Erwerbstätigkeit wiederum führt das zunehmende Alter zu einer erhöhten Wahrscheinlichkeit, aufgrund von eigener Krankheit oder Funktionseinschränkungen die Pflege des Partners oder der Partnerin nicht aufrechterhalten 
zu können. Auch hinsichtlich des Geschlechts können sich Unterschiede in den Bedarfslagen und beim resultierenden Unterstützungsbedarf ergeben. So gibt es viele Hinweise auf unterschiedliche, konkurrierende Sorgeverpflichtungen von Männern und Frauen ebenso wie auf unterschiedliche Ressourcen, die in die Pflege eingebracht werden können.

Betrachtet man häusliche Pflegearrangements vor dem Hintergrund der zugrundeliegenden Erkrankung des pflegebedürftigen Menschen, ergeben sich krankheitsspezifische Bedarfslagen. Angehörige von Menschen mit Demenz sind mit anderen Fragen in der Pflegesituation konfrontiert als Angehörige von Menschen nach einem Schlaganfall. Sowohl hinsichtlich des notwendigen Wissens und der Kompetenzen zur Bewältigung der Pflegesituation als auch hinsichtlich der Möglichkeiten zur Aufrechterhaltung der Beziehung zwischen pflegender und gepflegter Person ergeben sich daraus unterschiedliche Bedarfslagen.

Nicht zuletzt spielt die Frage der sozialen Beziehung zwischen pflegender und gepflegter Person eine Rolle. Kinder, die ihre Eltern oder Schwiegereltern pflegen, haben in der Regel andere, konkurrierende Verpflichtungen gegenüber Partnern, die füreinander sorgen. Für die Stabilität des Pflegearrangements ist es vor allem die Qualität der Beziehung, die einen wichtigen Einflussfaktor darstellt. Erfolgt die häusliche Pflege vor dem Hintergrund einer im Lebensverlauf gut harmonierenden Beziehung auf Basis gegenseitiger Sorge und Wertschätzung, so besteht darin eine wesentliche Ressource. Erfolgt die Versorgung jedoch vor dem Hintergrund langandauernder Spannungen und Auseinandersetzungen und fühlt sich der pflegende Teil der Beziehung eher gezwungen, die Pflege zu übernehmen, dann können sich diese Faktoren erschwerend auf das gesamte Arrangement auswirken.

Bedeutsam sind darüber hinaus die Entwicklungen in der Beziehung im Zeitverlauf. Häusliche Pflegearrangements sind nicht statisch, sondern entwickeln sich weiter. Der Verlauf der zugrundeliegenden Erkrankung, der weitere Verlust oder die Wiedererlangung der individuellen Selbständigkeit, die physische und psychische Belastung der Angehörigen, die unklare Prognose oder Probleme der Finanzierung der Situation stellen die handelnden Personen kontinuierlich vor Herausforderungen und erfordern regelmäßig neue Entscheidungen, z. B. bezogen auf die Inanspruchnahme von Unterstützungsleistungen hinsichtlich des Zeitpunkts, des Ausmaßes und des Inhalts.

Hinsichtlich der vorhandenen Bedarfslagen ergibt sich aus diesen nur kurz skizzierten Charakteristika häuslicher Pflegearrangements ein sehr komplexes Bild. Innerhalb von Familien und sozialen Netzwerken bedeutet die Übernahme von Pflege- und Sorgeverantwortung eine erhebliche Umstellung vorhandener Routinen, Rollen und Umgangsweisen. Gröning et al. (2015) haben die Pflege eines Familienmitglieds als „familiale Entwicklungsaufgabe“ bezeichnet. Der Bedarf besteht vorrangig darin, Unterstützung bei dieser Aufgabe durch Beratung und Begleitung zu erhalten, um den Aufgaben gewachsen zu sein, Kompetenzen dafür zu entwickeln und die notwendigen Entscheidungen treffen zu können. Zudem bedarf es für diese Aufgabe der notwendigen Zeit und Möglichkeiten zur Entlastung.

\subsection{Weiterentwicklung der Leistungen der Pflegeversicherung}

Wie hat sich nun das Leistungsspektrum der Pflegeversicherung, das diesen komplexen Bedarfslagen gerecht werden soll, im Laufe der Jahre entwickelt und in welche Richtung sollte es weiterentwickelt werden?

Der größte Teil der Ausgaben für Leistungen zur häuslichen Pflege entfällt auf die Leistungen nach $\$ \$ 36$ bis 38 SGB XI. Die Sachleistung nach $\$ 36$ SGB XI umfasst Leistungen, die durch einen zugelassenen ambulanten Pflegedienst im Haushalt des pflegebedürftigen Menschen erbracht werden. Art und Inhalt der Leistungen stehen in enger Verbindung mit dem Begriff der Pflegebedürftigkeit und wer- 
den in Rahmenverträgen nach $₫ 75$ SGB XI zwischen den Landesverbänden der Pflegekassen und den Verbänden der Leistungserbringer im Land konkretisiert. Entsprechend dem alten Begriff von Pflegebedürftigkeit wurden für die ambulante Pflege in erster Linie Leistungskomplexe mit Bezug zu den Alltagsverrichtungen in den Bereichen Körperpflege, Mobilität und Ernährung vereinbart. Mit der Einführung des neuen Begriffs der Pflegebedürftigkeit 2017 hat sich auch $\$ 36$ SGB XI dahingehend geändert, dass die Leistungen sich auf Beeinträchtigungen der Selbständigkeit in $\$ 14$ SGB XI - also den Aktivitäten und Lebensbereichen, die maßgeblich für die Feststellung der Pflegebedürftigkeit sind - beziehen sollen. Eine entsprechende Veränderung in den Rahmenverträgen zur ambulanten Pflege steht mehr als drei Jahre nach der Gesetzesänderung zum Zeitpunkt der Erstellung dieses Beitrags (Februar 2020) noch aus.

Die Geldleistung nach $₫ 37$ SGB XI ist vorgesehen für selbst beschaffte Pflegehilfen, um die erforderliche Pflege sicherzustellen. Der Bezug des Pflegegeldes geht einher mit der Verpflichtung, eine Beratung zur Sicherung der Qualität der häuslichen Pflege durch einen anerkannten Pflegedienst, eine anerkannte Beratungsstelle oder eine durch die Pflegekassen beauftragte Person abzurufen. Nach Einführung der Pflegeversicherung haben sich mehr als $80 \%$ der leistungsberechtigten Personen im häuslichen Bereich für diese Leistung entschieden (BMG 2020). Zwar ist diese Zahl mittlerweile gesunken, sie stellt jedoch nach wie vor die häufigste Leistungsform dar. Wenig beachtet wurde bislang der mit der Geldleistung verbundene obligatorische Beratungsbesuch, der je nach Pflegestufe beziehungsweise Pflegegrad zwei- oder viermal im Jahr abgerufen werden muss. Erst 2018 ist es gelungen, für diese Besuche Empfehlungen zur Qualitätssicherung zu verabschieden (Qualitätsausschuss Pflege 2019). Mehr als zwanzig Jahre lang hat diese jährlich millionenfach stattfindende Leistung kein größeres Interesse hervorgerufen. Das präventive Potenzial und die Möglichkeiten, bereits frühzeitig Probleme in
Pflegehaushalten erkennen und Unterstützung anbieten zu können, wurde nicht genutzt.

Weitere Leistungen zur häuslichen Pflege bestanden im Anspruch auf Urlaubs- und Verhinderungspflege sowie auf Tages- und Nachtpflege in teilstationären Einrichtungen. Diese Leistungen sind vor allem darauf ausgerichtet, Entlastungsmöglichkeiten für Angehörige pflegebedürftiger Menschen zu schaffen. Ebenfalls zur Verbesserung der Situation von informell pflegenden Personen wurden Leistungen zur sozialen Sicherung der Pflegeperson eingeführt. Zur Erleichterung und Verbesserung der Pflege stehen darüber hinaus noch Ansprüche auf Pflegehilfsmittel sowie für wohnumfeldverbessernde Maßnahmen zur Verfügung.

Eine Übersicht über die Veränderungen der Leistungen seit Einführung der Pflegeversicherung (Bäcker 2020) zeigt, dass der Kern der Leistungen seit Einführung bestehen geblieben ist, jedoch vielfältige Weiterentwicklungen erfahren hat, insbesondere im Hinblick auf die Beratung. Erste größere Veränderungen ergaben sich durch das PflegeQualitätssicherungsgesetz (PQsG) ab 2002, durch das Regelungen zum internen Qualitätsmanagement, zur Personalausstattung und zur Stärkung der Verbraucherrechte durch eine verbesserte Beratung und Information eingeführt wurden. Durch das PflegeleistungsErgänzungsgesetz, das ebenfalls 2002 in Kraft trat, wurden erstmals Erweiterungen eingeführt, die verdeutlichten, dass der verrichtungsorientierte Pflegebedürftigkeitsbegriff und allein darauf aufbauende Leistungen nicht ausreichend sind, um der Vielfältigkeit der Bedarfslagen zu entsprechen. So wurde ein zusätzlicher Leistungsanspruch für Pflegebedürftige mit einem erheblichen Bedarf an Beaufsichtigung und Betreuung - damit waren vor allem Menschen mit kognitiven Beeinträchtigungen gemeint - eingeführt, es wurden Verbesserungen für die teilstationäre Pflege vorgenommen und es wurde die Entwicklung von Versorgungskonzepten und -strukturen für Menschen mit Demenz durch niedrigschwellige Betreuungsangebote und die Entwicklung von Modellprojekten gefördert. 
Sehr umfangreich waren die Veränderungen durch das Pflegeweiterentwicklungsgesetz 2008. Darin wurde einerseits zur Verbesserung der Vereinbarkeit von Erwerbstätigkeit und Pflege das Pflegezeitgesetz gesetzlich verankert, das es Arbeitnehmerinnen und Arbeitnehmern ermöglicht, für eine kurze oder längere Zeit bei Erhalt des Arbeitsplatzes der Arbeit fernzubleiben. Erstmals wurden somit Regelungen geschaffen, die dem Spannungsfeld zwischen Erwerbstätigkeit und Pflege, dem sich viele pflegende Angehörige gegenübersehen, etwas entgegensetzten. Darüber hinaus wurden bestehende Leistungen erweitert, umfangreiche Regelungen zur Qualitätssicherung eingeführt und die Beratung zur Stärkung der ambulanten Versorgung deutlich gestärkt. Dies geschah durch die Einführung eines Rechtsanspruchs auf Beratung nach $\$ 7$ a SGB XI und den parallel angestrebten Aufbau einer Pflegeinfrastruktur, der jedoch bundesweit sehr unterschiedlich umgesetzt wurde und somit eher für Intransparenz und Ungleichheit in der Beratungsinfrastruktur gesorgt hat, auch wenn es in einzelnen Bundesländern oder einzelnen Kommunen durchaus zur Entwicklung tragfähiger Unterstützungsmöglichkeiten gekommen ist. Der zusätzliche Leistungsanspruch für Menschen mit einem erheblichen Bedarf an Beaufsichtigung und Betreuung wurde weiterentwickelt $\mathrm{zu}$ einer Leistung für Menschen mit eingeschränkter Alltagskompetenz und erheblich ausgeweitet. 2011 wurden durch das Familienpflegezeitgesetz weitere Möglichkeiten zur besseren Vereinbarkeit von Erwerbstätigkeit und Pflege geschaffen.

Das Pflege-Neuausrichtungsgesetz stellte 2012 die Weichen zur Einführung des neuen Pflegebedürftigkeitsbegriffs, der dann im Zuge des Pflegestärkungsgesetzes II (2015) tatsächlich eingeführt wurde. Daneben brachten die Pflegestärkungsgesetze Leistungserhöhungen, die Einführung von Betreuungsleistungen und weitere Neuerungen mit sich.

\subsection{Erwartungen an zukünftige Unterstützungsmöglichkeiten durch die Pflegeversicherung}

Es hat sich gezeigt, dass seit Einführung der Pflegeversicherung das Leistungsspektrum zur Unterstützung häuslicher Pflegearrangements deutlich erweitert wurde. Dennoch besteht die Notwendigkeit weiterer Ergänzungen und Anpassungen, die im Folgenden kurz beschrieben werden sollen:

\section{- Beratung}

Angesichts der Komplexität der häuslichen Pflege kommt der Beratung eine entscheidende Bedeutung zu. Dabei geht es nicht nur um die Weitergabe von Informationen zu Leistungen der Pflegeversicherung, sondern es geht darüber hinaus um Beratung zu pflegefachlichen Fragen, zur Begleitung, um den Aufgaben der häuslichen Pflege gerecht zu werden, zur Beratung bei oftmals vorkommenden Konfliktsituationen in der Pflege und nicht zuletzt zur Pflegerechtsberatung. Angebote dafür sind vorhanden, aber bundesweit nicht in gleicher Anzahl und insgesamt nur schwer überschaubar. Die Idee des Ko-Piloten, die durch den Bevollmächtigten der Bundesregierung für Pflege (2020), eingebracht wurde, kann als Reaktion auf diese Situation angesehen werden. Grundsätzlich ist gegen diese Idee auch nichts einzuwenden, es sollte jedoch darauf geachtet werden, dass keine Doppelstrukturen entstehen, da die Einführung des Rechtsanspruchs auf Beratung durch $\$ 7$ a SGB XI der gleichen Intention gefolgt ist. Vor diesem Hintergrund wäre es sicher angezeigt, die bestehenden gesetzlichen Möglichkeiten besser auszugestalten und weiterzuentwickeln, bevor weitere Strukturen geschaffen werden. Zudem sollte die Kompetenz in ambulanten Pflegediensten, die Zugang zu vielen Haushalten haben, insgesamt besser genutzt werden. 
- - Leistungsspektrum ambulanter Pflegedienste

Das Leistungsspektrum der ambulanten Pflegedienste ist trotz des neuen Pflegebedürftigkeitsbegriffs und der Änderung von $₫ 36$ SGB XI immer noch auf Alltagsverrichtungen ausgerichtet. Diese Engführung war bereits unter dem alten Pflegebedürftigkeitsbegriff nicht nachzuvollziehen und ist es unter dem neuen noch viel weniger. Somit können fachlich sinnvolle pflegerische Maßnahmen nicht erbracht werden und stehen den Pflegehaushalten nicht zur Verfügung. Auch im Sinne der fachlichen Weiterentwicklung und der Möglichkeiten für Pflegekräfte, ihre Kompetenzen tatsächlich zum Einsatz bringen zu können, ist die Erweiterung dringend geboten. Vorschläge zur Erweiterung des Leistungsspektrums liegen vor (Wingenfeld und Büscher 2017) und haben bereits Eingang in die neuen Verfahren der Qualitätsprüfung und -darstellung gefunden. Es bedarf jedoch ihrer Umsetzung durch entsprechende Veränderungen in den Rahmenverträgen.

\section{- Neue Leistungsformen}

Ebenfalls durch den Bevollmächtigten für Pflege (2020) wurde der Vorschlag zur Einführung eines Pflegebudgets für eine selbstbestimmte Pflege und Betreuung sowie eines Entlastungsbetrags zur Sicherung der Pflege und Entlastung von Angehörigen unterbreitet. Beide Budgets sollen dazu dienen, Leistungen flexibler zu gestalten und die Übersichtlichkeit für pflegebedürftige Menschen und ihre Angehörigen zu erhöhen. Sie weisen damit in eine Richtung, die der Komplexität häuslicher Pflegearrangements, wie sie in diesem Beitrag skizziert wurden, eher gerecht zu werden vermag. Für die Weiterentwicklung und Umsetzung dieses Vorschlags sollte auf Erfahrungen zurückgegriffen werden, die bereits vor längerem mit der Einführung von Budgets gemacht wurden (Klie und Blinkert 2008). Auch die Möglichkeiten zur Integration in das persönliche Budget nach $\$ 29$ SGB IX sollten dabei berücksichtigt werden.

\section{- - Stärkung des gesamten Pflegearrangements}

Erst durch die Pflegestärkungsgesetze wurde erstmals explizit die Möglichkeit eingeräumt, dass Angehörige pflegebedürftiger Menschen neben den Leistungen zur sozialen Sicherung der Pflegeperson - Leistungen im Rahmen der Pflegeversicherung empfangen können. Vormals wurde ihnen eine eher instrumentelle Rolle zugeschrieben. Nach und nach setzt sich die Erkenntnis durch, dass die Stabilisierung und Unterstützung des gesamten Pflegearrangements erforderlich ist, um die häusliche Pflege zu stärken. Neben der gezielten Beratung von Angehörigen und den Möglichkeiten zur Verbesserung der Vereinbarkeit von Erwerbstätigkeit und Pflege bedarf es darüber hinaus der Entwicklung von Formen der verbesserten Zusammenarbeit von formeller und informeller Pflege.

\section{- - Subjektorientierte Qualitätssicherung}

Die Diskussion um Leistungen und Qualität der Pflegeversicherung betrachtete pflegebedürftige Menschen und ihre Angehörigen bislang vor allem als Objekte, die der Unterstützung bedürfen. Beachtet wird ihre Situation nach wie vor in der Regel erst, wenn sie formelle Unterstützungsangebote in Anspruch nehmen. Der größte Teil pflegebedürftiger Menschen bleibt somit zunächst einmal sich selbst überlassen. Ein Ansatz der subjektorientierten Qualitätssicherung (Klie und Büscher 2019) in der ambulanten Versorgung nimmt die individuelle Situation des pflegebedürftigen Menschen, die im Rahmen der Begutachtung zur Feststellung der Pflegebedürftigkeit durch die Medizinischen Dienste festgestellt wird, zum Ausgangspunkt frühzeitiger Unterstützung und Prävention und trägt dadurch dazu bei, das problematische Entwicklungen der De-Stabilisierung häuslicher Pflegearrangements durch Überlastung und Konflikte erkannt und Unterstützungsmöglichkeiten aufgezeigt und initiiert werden können. 


\section{- Bessere Verknüpfung von ambulanter Langzeit- und Primärversorgung}

Die Problemlagen pflegebedürftiger Menschen gehen in der Regel über den Fokus der Pflegeversicherung hinaus, da es oftmals (chronische) Erkrankungen sind, die ursächlich für die beeinträchtigte Selbständigkeit der Menschen ist. Entsprechend besteht auch ein Bedarf an gesundheitlicher Primärversorgung. Die Verknüpfung der Sektoren durch eine verbesserte Kooperation zwischen niedergelassenen Ärzten und ambulanten Pflegediensten und Beratungsstellen liegt somit sehr nahe. Bislang sind die Sektorgrenzen nur schwer überwindbar und Möglichkeiten der regionalen integrierten Versorgung werden nur unzureichend wahrgenommen.

\section{Literatur}

Bäcker G (2020) Chronologie gesetzlicher Neuregelungen Pflegeversicherung und Pflege 1998-2019, Sozialpolitik aktuell. http://www. sozialpolitik-aktuell.de/tl_files/sozialpolitikaktuell/_Politikfelder/Sozialstaat/Chronik_ Dauerbaustelle/Dauerbaustelle.pdf. Zügegriffen: 24. Febr. 2020

Bowers B (1987) Intergenerational caregiving: adult caregivers and their ageing parents. Adv Nurs Sci 9:20-31

Bowers B (1988) Family perceptions of care in a nursing home. Gerontologist 28:361-371

Bundesministerium für Gesundheit (2020) Zahlen und Fakten zur Pflegeversicherung (Stand 17. Februar 2020). https:// www.bundesgesundheitsministerium.de/ fileadmin/Dateien/3_Downloads/Statistiken/ Pflegeversicherung/Zahlen_und_Fakten/Zahlen_ und_Fakten_der_SPV_17.Februar_2020_barr.pdf. Zugegriffen: 26. Febr. 2020

Der Bevollmächtigte der Bundesregierung für Pflege (2020) Leistungsdschungel in der häuslichen Pflege auflösen. Diskussionspapier zum Entlastungsbudget. Der Bevollmächtigte der Bundesregierung für Pflege, Berlin

Gröning K, Sander B, von Kamen R (Hrsg) (2015) Familiensensibles Entlassungsmanagement. Mabuse, Frankfurt am Main

Klie T, Blinkert B (2008) Das Pflegebudget. Abschlussbericht 2008. https://www.gkv-spitzenverband.de/ media/dokumente/pflegeversicherung/forschung/ projekte_unterseiten/pflegebudget/1Anlage_PBGesamtzusammenfassung_3271.pdf. Zugegriffen: 26. Febr. 2020

Klie T, Büscher A (2019) Subjektorientierte Qualitätssicherung in der Langzeitpflege. Nachrichtend Dtsch Vereins Öffentliche Priv Fürsorge E V 99:114-119

Meyer JA (1996) Der Weg zur Pflegeversicherung. Positionen - Akteure - Politikprozesse. Mabuse, Frankfurt am Main

Nolan M, Grant G, Keady J (1996) Understanding family care. Open University Press, Buckingham, Philadelphia

Qualitätsausschuss Pflege (2019) Empfehlungen zur Qualität der Beratungsbesuche nach $\S 37$ Abs. 5 SGB XI. https://www.gs-qsa-pflege.de/dokumentezum-download/. Zugegriffen: 26. Febr. 2020

Statistisches Bundesamt (2003) Bericht: Pflegestatistik 2001. Pflege im Rahmen der Pflegeversicherung - Deutschlandergebnisse. Statistisches Bundesamt, Zweigstelle, Bonn

Statistisches Bundesamt (2018) Pflegestatistik 2017. Pflege im Rahmen der Pflegeversicherung - Deutschlandergebnisse. Statistisches Bundesamt, Zweigstelle, Bonn

Twigg J, Atkin K (1994) Carers perceived: policy and practice in informal care. McGraw-Hill, New York

Wingenfeld K, Büscher A (2017) Strukturierung und Beschreibung pflegerischer Aufgaben auf der Grundlage des neuen Pflegebedürftigkeitsbegriffs. Fachbericht im Auftrag des Bundesministeriums für Gesundheit. Bielefeld/Osnabrück. https://www.bundesgesundheitsministerium. de/fileadmin/Dateien/5_Publikationen/Pflege/ Berichte/Fachbericht_Pflege.pdf. Zugegriffen: 26 . Febr. 2020 
Open Access Dieses Kapitel wird unter der Creative Commons Namensnennung 4.0 International Lizenz (http:// creativecommons.org/licenses/by/4.0/deed.de) veröffentlicht, welche die Nutzung, Vervielfältigung, Bearbeitung, Verbreitung und Wiedergabe in jeglichem Medium und Format erlaubt, sofern Sie den/die ursprünglichen Autor(en) und die Quelle ordnungsgemäß nennen, einen Link zur Creative Commons Lizenz beifügen und angeben, ob Änderungen vorgenommen wurden.

Die in diesem Kapitel enthaltenen Bilder und sonstiges Drittmaterial unterliegen ebenfalls der genannten Creative Commons Lizenz, sofern sich aus der Abbildungslegende nichts anderes ergibt. Sofern das betreffende Material nicht unter der genannten Creative Commons Lizenz steht und die betreffende Handlung nicht nach gesetzlichen Vorschriften erlaubt ist, ist für die oben aufgeführten Weiterverwendungen des Materials die Einwilligung des jeweiligen 4 Rechteinhabers einzuholen. 